\title{
A Wide-Angle View Endoscope System Using Wedge Prisms
}

\author{
Etsuko Kobayashi ${ }^{1}$, Ken Masamune ${ }^{2}$, Ichiro Sakuma ${ }^{1}$ and Takeyoshi Dohi ${ }^{1}$ \\ ${ }^{1}$ Institute of Environment Studies, Grad. School of Frontier Sciences, \\ The University of Tokyo,7-3-1 Hongo Bunkyo-ku, Tokyo, 113-8656, Japan \\ \{etsuko, sakuma, dohi\}@miki.pe.u-tokyo.ac.jp \\ ${ }^{2}$ Depatment of Bio-technology, Tokyo Denki University, \\ Ishizaka, Hatoyama-cho, Saitama, 350-0394, Japan \\ masa@b.dandai.ac.jp
}

\begin{abstract}
We describe a novel robotic endoscope system. It can be used to observe a wide area without moving or bending the endoscope. The system consists of a laparoscope with zoom facility and two wedge prisms at the tip. This new concept produces excellent characteristics as follows. Firstly, it can change the field of view even in a small space. Secondly, it is safe because it avoids the possibility of hitting internal organs. Finally, because it does not require a large mechanism for manipulation of the endoscope, it does not obstruct the surgeon's operation. During evaluation, we confirmed that the range of view and levels of image deformation were acceptable for clinical use.
\end{abstract}

\section{Introduction}

Endoscopic surgery is becoming increasingly popular as a form of minimally invasive surgery. In such surgery, smooth manipulation of the endoscope is essential. However, it is difficult for surgeons and camera assistants to manipulate the endoscope steadily and smoothly, because of the restricted space and degrees of freedom of movement, and because of limitations in hand-eye coordination.

Robotic endoscopic systems have the potential to solve these problems [1][2][3]. They can hold the endoscope steadily and the surgeon can exert remote control using a computer display. For these reasons, many robotic endoscopic systems have been developed. Most of them manipulate existing endoscopes, of which there are two types: rigid and flexible. Rigid endoscopes need to rotate the insertion point to observe a wide range of views, whereas flexible types rotate the tip of the endoscope to achieve the same end. Thus, to obtain a wide field of view a large operating area is required.

However, in endoscopic fields such as neurosurgery, where there is normally only a limited operating area, it is difficult to obtain a sufficiently wide field of view. It is therefore desirable to develop a new type of endoscope that can achieve a wide range of views without moving the endoscope itself.

There is a commercially available laparoscopic system that alters the field of view by trimming the field viewed by the laparoscope and moving the cutting tool area appropriately [4]. With this system, the surgeon can move the field of view without moving the endoscope. However, because the image uses only part of the whole, the quality is poor. 
With this in mind, we propose a new type of robotic wide-view endoscope that does not require rotation or bending of the endoscope to move the field of view. This is clinically significant as it allows the surgeon to make extensive observations in a small space and is safe because it avoids the possibility of accidentally hitting organs.

In Section 2, we propose a design for a novel robotic endoscope, with wedge prisms for moving the field of view. Theoretical formulae for controlling the image are presented in Section 3. In Section 4, we validate the basic specification of this endoscope system.

\section{System Description}

\subsection{System Requirements}

To develop a first prototype of a wide-view endoscope system, we decided to focus on the rigid type laparoscope, since it is ubiquitous in minimally invasive surgery and provides high quality images.

The requirements of this system are as follows.

Movement of the laparoscopic view

In laparoscopic surgery, the laparoscope should be able to rotate around the insertion point and to move back and forth. Thus, the wide-view laparoscope should have at least two degrees of angular freedom and be able to zoom in and out.

Sterilization

Because the tip of the endoscope is inserted in the abdomen, it needs to be sterilized. To achieve this, the mechanism must be simple.

High quality image

As for all medical endoscopes, the image quality must be good.

\subsection{System Description}

To achieve the above requirements, we used two wedge prisms and a laparoscope with zoom capacity. Figure 1 depicts a wedge prism. It has a vertex angle of $\left(\theta_{\mathrm{w}}\right)$ to bend the light axis $\theta_{\mathrm{d}}$. The relationship between $\theta_{\mathrm{d}}$ and $\theta_{\mathrm{w}}$ is as follows:

$$
\theta_{w}=\arctan \left[\frac{\sin \theta_{d}}{n-\cos \theta_{d}}\right]
$$

where ' $n$ ' is the refractive index of the prism (Fig. 1a).

If the wedge prism is put in the tip of a laparoscope and rotated around its axis, the light axis turns around the axis with an angle of $\theta_{\mathrm{d}}$ (Fig. 1b).

Another wedge prism that has the same wedge vertex angle $\left(\theta_{\mathrm{w}}\right)$ as the first is mounted close behind the first (Fig. 1c). When the two prisms are rotated around the light axis of the laparoscope, the light axis can bend to any direction in a cone. If the wedge vertex angle $\left(\theta_{\mathrm{w}}\right)$ is small, the maximum angle of such bending is $2 \theta_{d}$ (Fig. $1 \mathrm{a}$ ). 
(a)

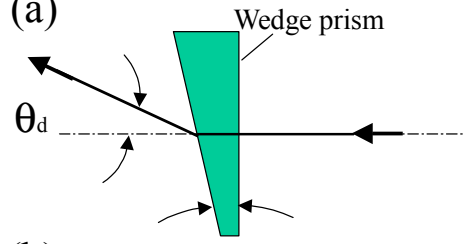

(c)

(b)

$\theta_{\mathrm{w}}$
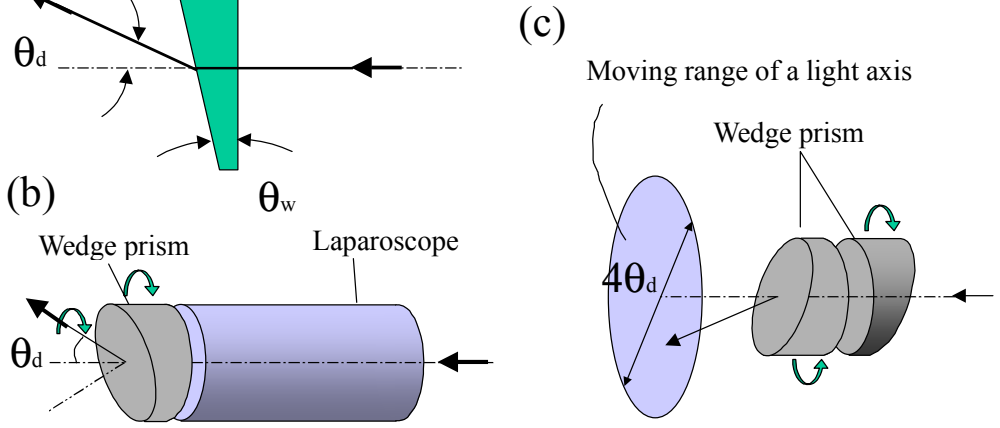

Fig.1. Wedge prism

a) Cross-section of a wedge prism. The wedge prism has a wedge vertex angle $\left(\theta_{\mathrm{w}}\right)$ to refract light at an angle of $\theta_{\mathrm{d}}$; b) Laparoscope with a wedge prism; c) Combination of two wedge prisms.

Figure 2 shows the construction of the wide-view endoscope system. We used an automatic micro-zoom laparoscope (Shinko Optical Co. Ltd, Tokyo, Japan). It consists of a short $(155-\mathrm{mm})$ laparoscope with one lens at its tip, an automatic zoom component and a charge coupled device (CCD) camera[5]. Two wedge prisms are mounted in the tip, and each prism is set on a sleeve. These two sleeves rotate independently around within the laparoscope, and are connected to motors by gears. The system is controlled by the surgeon, using a man-machine interface.

The wedge vertex angle of the wedge prism that we used was $18^{\circ} 8^{\prime}$ and the light bending angle $\left(\theta_{\mathrm{d}}\right)$ was $10^{\circ}$. Therefore, the system can bend the light axis in a cone with a vertex angle of $40^{\circ}$. In this mechanism, only two sleeves rotate within the laparoscope, so the mechanism can be simple and small, and yet can observe a wide range of view.

Figure 3 shows a manual version of the wide-view endoscope. The diameter of the prism was $25 \mathrm{~mm}$. We are constructing a smaller motorized prototype with a smaller (12-mm) prism.

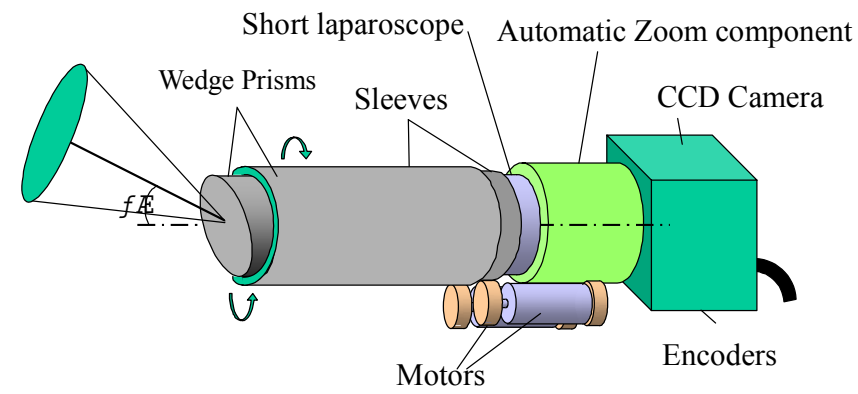

Fig.2. Construction of the wide-range endoscope system 


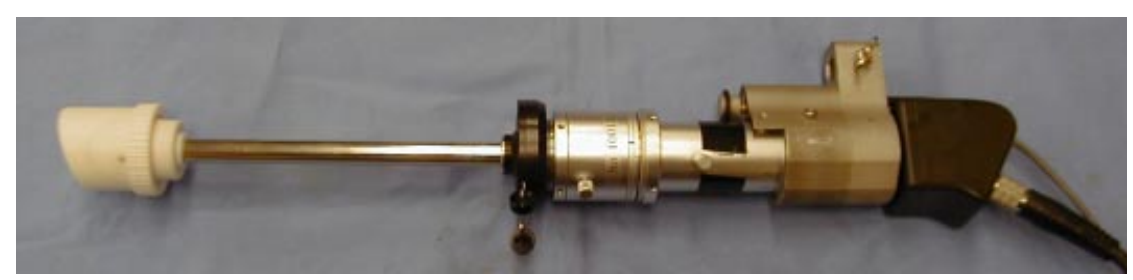

Fig.3. Manual type of wide-view laparoscope

\section{Controlling the Image}

Figure 4a shows the coordinates and parameters we used. The wedge prism that is nearest the tip of the laparoscope is named Prism 1. The other prism is named Prism 2. Figure $4 \mathrm{~b}$ shows a cross-section in the $\mathrm{YZ}$ plane. The origin is set at a node of the light axis and Prism 1. As we decided that positioning accuracy was not required for the final endoscopic image, we used approximate values for some parameters and we considered the optical model to be that of a simple pinhole camera model. The distance between the virtual screen and the focal point is $Z_{f}$. The distance between the virtual screen and the origin is $Z_{1}$ and the distance between the virtual screen and the node is $Z_{2}$. In this model, because of limited space in the abdomen, we assumed that all the targets were in the virtual screen plane.

Figure $4 \mathrm{~b}$ shows the refracting angle of each prism. For Prism 2, to simplify the formula, we assumed that the angle of incidence (equal to the refracting angle of Prism 1) was zero degrees [6].

$$
\begin{aligned}
& \phi_{1}=\arcsin \left\{n \sin \left(D\left(\theta_{w}\right)\right\}\right. \\
& \phi_{2}=D\left(\theta_{w}\right) \\
& D\left(\theta_{w}\right)=-\theta_{w}+\arcsin \left(n \sin \theta_{w}\right)
\end{aligned}
$$

The relation between the target point and the rotation angle of the prisms is shown in Fig. 4c. In the virtual screen, the reflected light axis of Prism 1 appears as a circle with a radius of $R_{1}$. The reflected light beam of Prism 2 is a circle with a radius of $R_{2}$.

The reflected direction is decided by the rotation angle of each prism $\left(\theta_{1}, \theta_{2}\right)$. Therefore, the target point vector, $\mathbf{p}(\mathrm{Px}, \mathrm{Py})$, was found as follows:

$$
R_{1}=Z_{1} \sin \phi_{1}, R_{2}=Z_{2} \sin \phi_{2}
$$

$\mathbf{p}=\mathbf{r}_{1}+\mathbf{r}_{2}$, where $\mathbf{r}_{1}$ is a vector with length $R_{1}$ and an angle with the $X$ axis of $\theta_{1}$, and $\mathbf{r}_{2}$ is a vector with length $R_{2}$ and an angle with the $X$ axis of $\theta_{2}$.

The angle of each prism is calculated as follows (Fig. $4 \mathrm{~d}$ ).

$$
\begin{aligned}
& \theta_{1}=\theta_{a}+\theta_{b}=\arctan \left(\frac{P_{y}}{P_{x}}\right)+\arccos \left(\frac{\sqrt{P_{x}^{2}+P_{x}^{2}}}{2 \cdot R_{1}}\right) \\
& \theta_{2}=\arctan \left(\frac{P_{y}-R_{1} \sin \theta_{1}}{P_{x}-R_{1} \cos \theta_{1}}\right)
\end{aligned}
$$


(a)

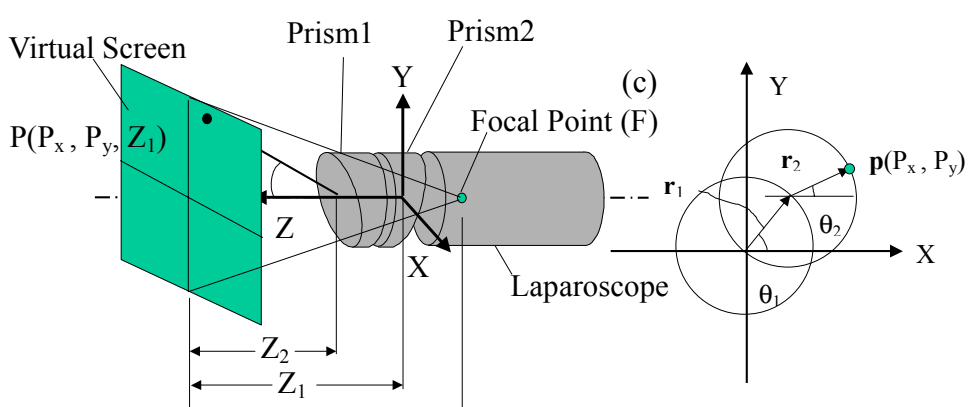

(b)

(d)

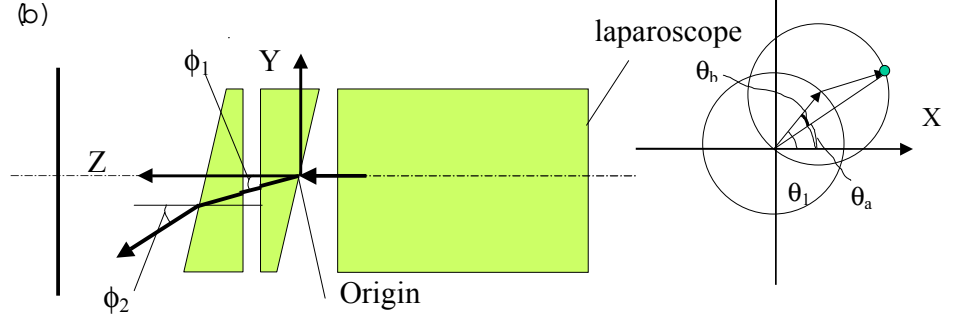

Fig. 4. Controlling the image, (a) Optical model of the wide-view laparoscope; (b) Cross-section of the system; (c) Virtual screen; (d) Virtual screen.

\section{Evaluation}

\subsection{Measurement of the Range of View}

Firstly, we measured the range of view of the endoscope. Figure 5 shows the arrangement of the experiment. The test screen was set $5.4 \mathrm{~cm}$ in front of the tip of the laparoscope. We measured the center points (the node of the light axis and the screen) of the highest, the lowest, and the extreme left and right views. As a result, the range of view was $41^{\circ}$ in height and $39^{\circ}$ in width. Figure 6 shows the highest and lowest endoscopic images of a life-size liver model. The liver model was set $7.5 \mathrm{~cm}$ in front of the tip. You can see that the surgeon can observe the almost whole liver by the wide-view endoscope. 


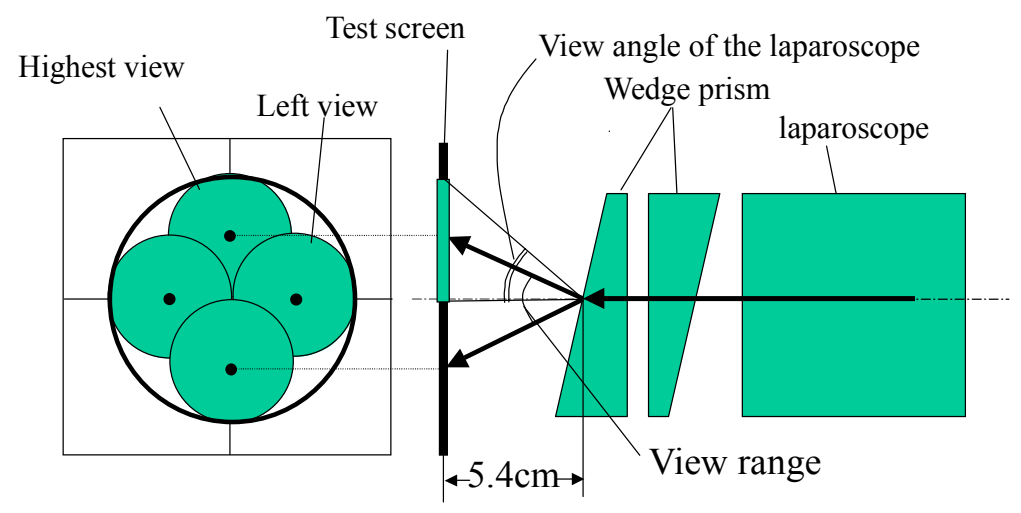

Fig.5. Arrangement of the experiment

Left is a front view of the test screen. Right is a side view of the experimental arrangement.

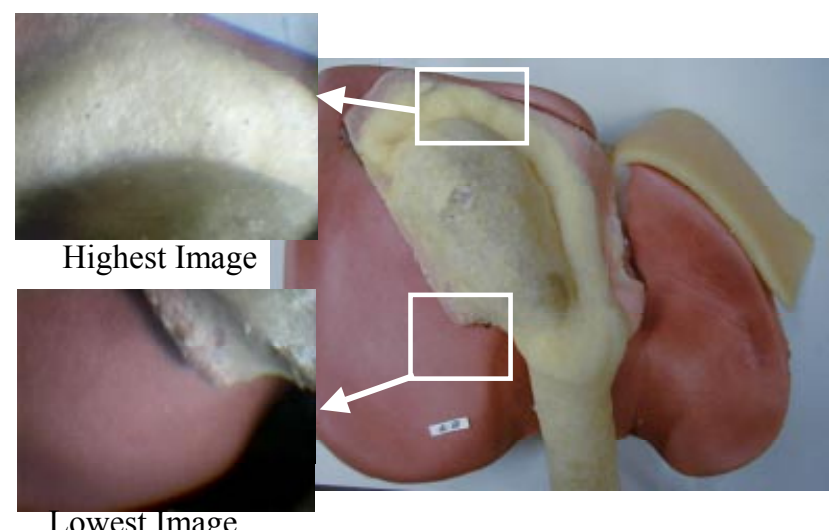

Lowest Image

Fig.6. Wide-view endoscopic image of a life-size liver model

\subsection{Evaluation of Image Deformation}

We evaluated the image deformation caused by the prisms. Table 1 shows the ratio of the height to the width of a 5-mm square. We measured this for the highest, lowest, and extreme left and right images. For comparison, we measured the deformation of the periphery of the normal laparoscopic image. 
Table 1. Comparison of deformation of the image between the experimental system and the unaltered laparoscope.

\begin{tabular}{|l|l|l|}
\hline \multirow{3}{*}{ Wide-view laparoscope } & Images & Ratio of height to width \\
\hline \hline \multirow{2}{*}{} & Highest image & 0.85 \\
\cline { 2 - 3 } & Lowest image & 0.98 \\
\cline { 2 - 3 } & Left end & 0.97 \\
\cline { 2 - 3 } & Right end & 1.05 \\
\hline Ordinary laparoscope & & 1.02 \\
\hline
\end{tabular}

\section{Discussion}

In this system, the surgeon can move the field of view without rotating or bending the endoscope. Therefore, this system offers excellent advantages.

-It can move the field of view, even in a small space.

-It is completely safe because it also avoids the possibility of hitting organs.

-Because it does not require a large mechanism for moving the endoscope, it does not obstruct the surgeon's operation.

In the evaluation, we confirmed the wide range of movement of our system. Because the view angle of the laparoscope is $60^{\circ}$, we can observe a circle with a diameter of $17 \mathrm{~cm}$ when only $5 \mathrm{~cm}$ in front of the tip of the endoscope. By contrast, the ordinary laparoscope can only observe a circle of $5.6 \mathrm{~cm}$ without being moved. From this result, for most of the organs the whole structure can be observed without moving the endoscope. Therefore, we can say it has a sufficiently wide range for clinical use.

There was some deformation of the image. However, it was considered acceptable, because it was similar to that seen in the periphery of the ordinary laparoscopic image. Color aberration is also inevitable because of the prisms. These aberrations and deformations could be simply corrected by an image processing board.

\section{Conclusions}

We propose a new type of endoscope system that can allow observation over a wide angle and provide high quality images without moving the endoscope itself. This has great significance for future clinical use.

\section{Acknowledgement}

This study was partly supported by the Research for the Future Program (JSPS-RFTF 96P00801) and (JSPS-RFTF 99I00904) 


\section{References}

1. Taylor RH, Funda J, Eldridge B, Gomory S, Gruben K, LaRose D, Talamini M, Kavossi L, Anderson J A Telerobotic Assistant for Laparoscopic Surgery. Computer Integrated Surgery: The MIT Press, (1995) pp 581-592

2. Sackier JM, Wang Y Robotically Assisted Laparoscopic Surgery: From Concept to Development. Computer Integrated Surgery: The MIT Press, (1995) pp 577-580

3. Finlay PA, Ornstein MH Controlling the Movement of a Surgical Laparoscope. IEEE Engineering in Medicine and Biology Vol.14 No.3 (1995) pp.289-299

4. Olympus co.ltd. Home page, http://www.olympus.co.jp/

5. Etsuko Kobayashi, Ken Masamune, Ichiro Sakuma, Takeyoshi Dohi, Daijo Hashimoto A New Safe Laparoscopic Manipulator System with a Five-Bar Linkage Mechanism and an Optical Zoom Journal of Computer Aided Surgery, 4(4), (1999) pp.182-192

6. Faugeas:Three-Dimentional Computer Vision, MIT Press, 1993 